\title{
Early indications that low mental quality of life scores in recently unwell older people predict downstream functional decline
}

This article was published in the following Dove Press journal:

Clinical Interventions in Aging

10 April 2015

Number of times this article has been viewed

\author{
Alvin Atlas \\ Karen Grimmer \\ Kate Kennedy \\ International Centre for Allied \\ Health Evidence, University of South \\ Australia, Adelaide, SA, Australia
}

Background: Accurately detecting markers of early functional decline (FD) are essential to support older people to successfully age in place; however, these markers are poorly understood. We tested the hypothesis that compromised mental quality of life after a minor health crisis could be an early predictor of FD.

Methods: This longitudinal observational cohort study was conducted in the emergency department (ED) of a large Australian hospital and in the community. Data were collected from 148 community-dwelling people aged $65+$ years, who provided data at recruitment (baseline), and at 1 month and 3 months post discharge from the ED. Short Form-12 mental quality of life component scores (MCS) were regressed with patient descriptors taken at baseline (age, sex, socioeconomic status, education, Mini-Mental State Examination, and primary language), and over-time estimates of FD taken at baseline, and at 1 and 3 months post discharge (instrumental activities of daily living, frequency of falls and hospitalizations, use of gait aids, receipt of community services, living status, and requiring a carer).

Results: MCS at 1 month (MCS1) post ED discharge was significantly associated with instrumental activities of daily living at $1(r=0.45, P<0.001)$ and 3 months $(r=0.401, P=0.001)$ post ED discharge, but not at baseline $(r=0.010, P>0.05)$. Subjects with lower than the population median MCS showed a significant linear decline in total instrumental activities of daily living scores over 3 months $(P=0.025)$. There was no linear trend over time in the relationship between MCS1 with frequency of falls $(P=0.20)$ or hospitalizations $(P=0.42)$; however, there was a significant difference at 3 months post ED discharge for falls $(P=0.036)$ and hospitalizations $(P=0.039)$ between low and high MCS1 groups. There were no significant confounders.

Conclusion: Low MCS scores 1 month after a minor health crisis appear to significantly predict downstream FD. This finding needs to be tested in a larger sample.

Keywords: instrumental activities of daily living, mental quality of life, functional decline, falls, hospitalization

\section{Introduction}

The world's population, especially in developed countries, is rapidly aging. ${ }^{1}$ In Australia, the proportion of the population aged 65 years and over has almost doubled in the last 40 years, from $8.3 \%$ in 1971 to $14 \%$ in 2011 (reflected as 3.22 million Australians in this age group). ${ }^{2}$

Physical and mental aging processes in body systems are associated with functional decline (FD). FD is complex and currently poorly understood, but is associated with being less able to live safely and independently in the community. ${ }^{3-5}$ Given the increasing number of older people who will be living in Australian communities within the next 5 years, it is critical to better understand how FD first manifests, and to identify early
Correspondence: Alvin Atlas International Centre for Allied Health Evidence (iCAHE), University of South Australia, City East Campus, Playford, GPO Box 247I, Adelaide, Australia 500I $\mathrm{Tel}+6 \mid 883021800$

Fax +61883022853

Email alvin.atlas@unisa.edu.au 
triggers for FD, so that effective interventions can be put in place to arrest or slow its trajectory. ${ }^{4,6,7}$ It seems that once an individual is well established on a FD trajectory, it is difficult to reverse the progression of decline. ${ }^{8}$ Commonly reported measures of established FD in community-dwelling older people include loss of independence in basic activities of daily living or instrumental activities of daily living (IADL), increased frequency of falls and/or hospitalizations, increased need for home assistance, increasing use of gait aids, loss of morale, and onset of depression and/or anxiety. ${ }^{9-12}$

There is no agreement on a comprehensive, efficient battery of FD measures, nor on the best timing for assessment or reassessment. Most commonly, assessments for FD are made when an older person comes to the attention of health providers (usually in a health crisis) at an emergency department (ED) presentation or hospital admission. This timing is generally too late for effective intervention to regain independent, safe community living. ${ }^{7,11}$ Common causes of ED admission among the elderly have been reported to impairment of general condition, dyspnea, falls and trauma, abdominal problems, thoracic pain, syncope, dizziness, and minor stroke. ${ }^{9}$

Quality of life is not commonly reported as an associate of FD, although it seems reasonable to assume that compromised quality of life and FD could be biologically linked. Decreasing physical activity has been associated with increasing frailty, declining function, compromised cognition, and poor mental health. ${ }^{8,11,13,14}$ Biologically, compromised mental health could equally be a precursor of declining physical function, as the direction of association has not been well researched. ${ }^{7}$

This paper tests the hypothesis that compromised mental quality of life in older people who have suffered a recent minor health crisis could be an early predictor of FD. For instance, if older people start to lose confidence, and doubt their capacity to manage safely and independently in the community following a minor health event for which they presented to ED, it is plausible that this may be subsequently reflected in reduced physical activity and function, increased falls, or increased need for a carer or gait aid. This could lead to consequences of increased use of health care services and/ or earlier than preferred transition to residential care. ${ }^{11}$

This paper attempted to estimate the impact of low mental quality of life scores on the trajectory of FD over a 3-month period in community-dwelling older people who presented at a hospital ED for a minor health complaint that did not result in a hospital admission.

\section{Materials and methods}

This was a longitudinal observational cohort study of older community-dwelling people with minor health crises and aged $65+$ years who were admitted to and discharged from the ED of one large public tertiary Australian hospital. Minor health crisis is defined as non-life-threatening health situations presented by older individuals at ED presentation, generally not requiring hospital admission (ED triage categories 4 and 5). ${ }^{15}$ We previously reported comprehensive details on sample recruitment and demographics. ${ }^{3}$ Subjects were enrolled between September 2011 and February 2012, and data collection ceased in April 2012. We attempted to collect data from subjects at three time points, ie, recruitment (baseline, when measures were taken face-to-face), and at 1 and 3 months after discharge from ED (by telephone interview).

Subjects were eligible to participate if they were 65 years of age or more, provided informed consent, were able to understand spoken English and had sufficient hearing to be contacted by telephone, and were not admitted to a hospital ward for the index presentation with which they presented and were subsequently discharged from ED.

Consenting subjects remained eligible for the study sample after they were discharged from ED, if they were not subsequently admitted to hospital as a result of their index ED presentation, if they were not subsequently admitted to residential care, and if they were contactable by telephone at the follow-up time points. Ethical approval was provided by the human research ethics committees of the authors' university and the participating hospital.

\section{Measures}

At recruitment (baseline), subjects provided information on immutable factors (age, sex, socioeconomic status) collected as "Socio-Economic Indexes for Areas (SEIFA) Index of Relative Socio-economic Disadvantage (IRSD)", ${ }^{16}$ and the highest level of education and primary language at home. Subjects also provided information on potentially mutable indicators of FD (community services support, gait aids, personal carer, living status, numbers of falls and hospitalizations during the previous 6 months, and IADL over the past 1-2 days). ${ }^{17,18}$ A total IADL score was derived from the sum of individual IADL domains, ${ }^{17}$ and this score was used for the analysis reported in this paper. The Mini-Mental State Examination (MMSE) ${ }^{19}$ was administered at baseline.

At 1 and 3 months' telephone follow-up, eligibility and consent was first confirmed, after which all mutable variables and standard FD outcome measures were remeasured. 
The MMSE was not reassessed because it could not be viably administered over the telephone. At both follow-up time points, quality of life was measured using the Short-Form 12 (SF12) ${ }^{20,21}$ This is an internationally used measure of physical and mental quality of life, from which individual scores can be compared with age-sex population norms. ${ }^{22,23}$ The SF12 estimates an individual's health-related quality of life and functioning across eight conceptual measures (physical functioning, role limitations due to physical health, emotional problems and mental health, bodily pain, general health, vitality, and social functioning). The physical (PCS) and mental (MCS) components of the SF12 are commonly used in clinical and epidemiological research. ${ }^{24}$ The component physical and mental scores were generated from the SF12 scoring template. ${ }^{25}$

\section{Research questions}

This paper reports on four research questions in which the relationship between MCS measured at 1 month post ED discharge was determined, with other data collected at one or more of the three time points (Figure 1):

1. Is MCS at 1 month related to the immutable baseline measures and mutable measures of FD at baseline and 1 and 3 months' post ED discharge?

2. What is the correlation between MCS at 1 month, and change in each mutable FD measure between baseline and 1 month post ED discharge?

3. What are the associations between MCS at 1 month and FD measures also measured at 1 month post ED discharge?

4. What is the predictive capacity of MCS at 1 month for FD measures measured 3 months post ED presentation?

\section{Data management}

Microsoft $\mathrm{Excel}^{26}$ was used to record all data. For this paper, raw MCS and PCS scores were standardized using the scoring template, and categorized using the relevant median age-sex population scores into low and high scores.

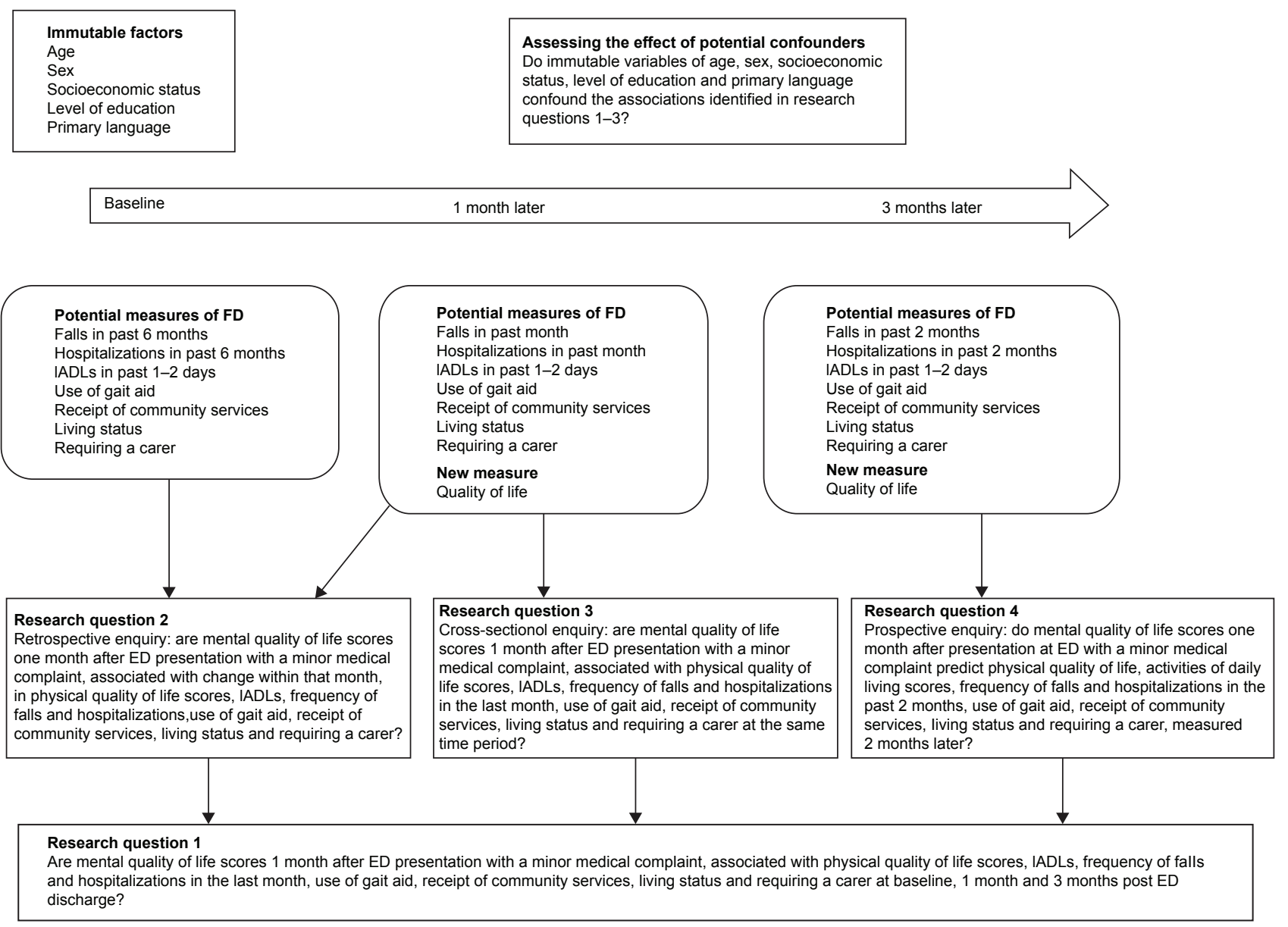

Figure I Research questions and time line of data collection.

Abbreviations: ED, emergency department; FD, functional decline; IADL, instrumental activities of daily living. 
Considering the immutable baseline variables, age was analyzed in three categories of $65-74,75-84$, and $85+$ years; sex and primary language at home were reported in binary form (language as English or not); education was analyzed as "primary school only", "primary and any secondary schooling", or "any tertiary education"; and socioeconomic status was calculated initially into ten SEIFA categories, which were collapsed into five by combining adjacent categories. The lowest SEIFA category (the two poorest socioeconomic status classifications) was used as the default comparator for analysis. MMSE scores were considered as continuous data and as categories (data split at its median). Mutable FD data was reported as per month rates of falls and hospitalization; total IADL scores (presented as continuous data, and also cut at the median value); and binary data on use of gait aid, receipt of community services, requiring a carer, and living alone at home.

\section{Data analysis}

Stata version 12.1 (StataCorp, College Station, TX, USA) ${ }^{27}$ was used for all analysis. Descriptive statistics, stratified by high/low MCS at 1 month, were generated for all study variables. To interpret changes from baseline and 1 month: for IADL total score, an increase in the total score over time was categorized as improved function; for frequency of hospitalization and falls, an increase over time was categorized as decreased function. Significance was determined by $P$-values $<0.05$. Univariate logistic regression models were applied to determine the crude associations relevant to each research question, reported as odds ratios and 95\% confidence intervals. Significant associations were determined when the 95\% confidence intervals did not encompass 1 . The confounding influence of the immutable variables and baseline MMSE scores on the association between MCS at 1 month and FD measures in questions 2-4 was tested using stepwise multivariate logistic regression models, with significant change in the log likelihood ratio $(P<0.05)$ used to identify significant adjustment in the association with the introduction of each immutable variable. Trend analysis was undertaken to determine the significance of linear trends over time and to estimate the trajectory of the mutable FD measures over time, considering each FD measure and MCS status at 1 month.

\section{Results}

\section{Subjects}

This paper reports on our analysis of a subset of the total recruited sample, this being the 148 subjects who provided complete data at all three time points (baseline, 1 month, and
3 months post ED discharge) for all variables of interest. The consort diagram reporting subject recruitment and attrition over the study period is provided in Figure 2.

\section{Association between immutable baseline measures and mutable measures of FD at months I and 3}

Considering study question 1 , Table 1 reports the differences between high and low categories of mental quality of life component score at 1 month (MCS1) in immutable variables measured at baseline, MMSE measured at baseline, and the mutable measures of FD measured at baseline and 1 and 3 months post ED discharge. Subjects with low MCS1 had significantly lower total IADL scores, more falls, and greater use of gait aids than those with high MCS1 at most time points. There was no difference in MMSE scores between the two MCS1 groups, suggesting that cognitive function at $\mathrm{ED}$ presentation was not related to downstream mental quality of life.

Table 2 reports the change between measures taken at baseline and 1 month after ED presentation. Change is reported as scores remaining the same, scores improving, or getting worse over time. Although the frequency of improved total IADL score was greater than the frequency of decreased IADL score, the mean change in IADL score was not significant $(0.263, P=0.56)$. There was also a decrease in the number of subjects with falls and hospitalization between baseline and 1 month retest. One month after ED presentation, there is an increase in the number of subjects who require a carer $(14 \%)$, with those not requiring a carer anymore increasing by $8 \%$. For living status (now living with someone), use of gait aids (now uses gait aids), and receipt of community support (now receiving support), the changes from baseline to 1 month after ED presentation were $22 \%$, $30 \%$, and $25 \%$, respectively. The results also showed that in all of the measures, no change from baseline to 1 month was evident. The short time span between measurements may influence the result or change.

\section{Association between mutable FD measures and MCSI scores}

Considering study question 2, there was no significant association between MCS1 scores and amount of change in the mutable FD measures between baseline and 1 month (Table 3).

\section{Association between MCSI and FD measures measured at month I}

For study question 3, only the total IADL score at 1 month post ED discharge was significantly associated with MCS1 


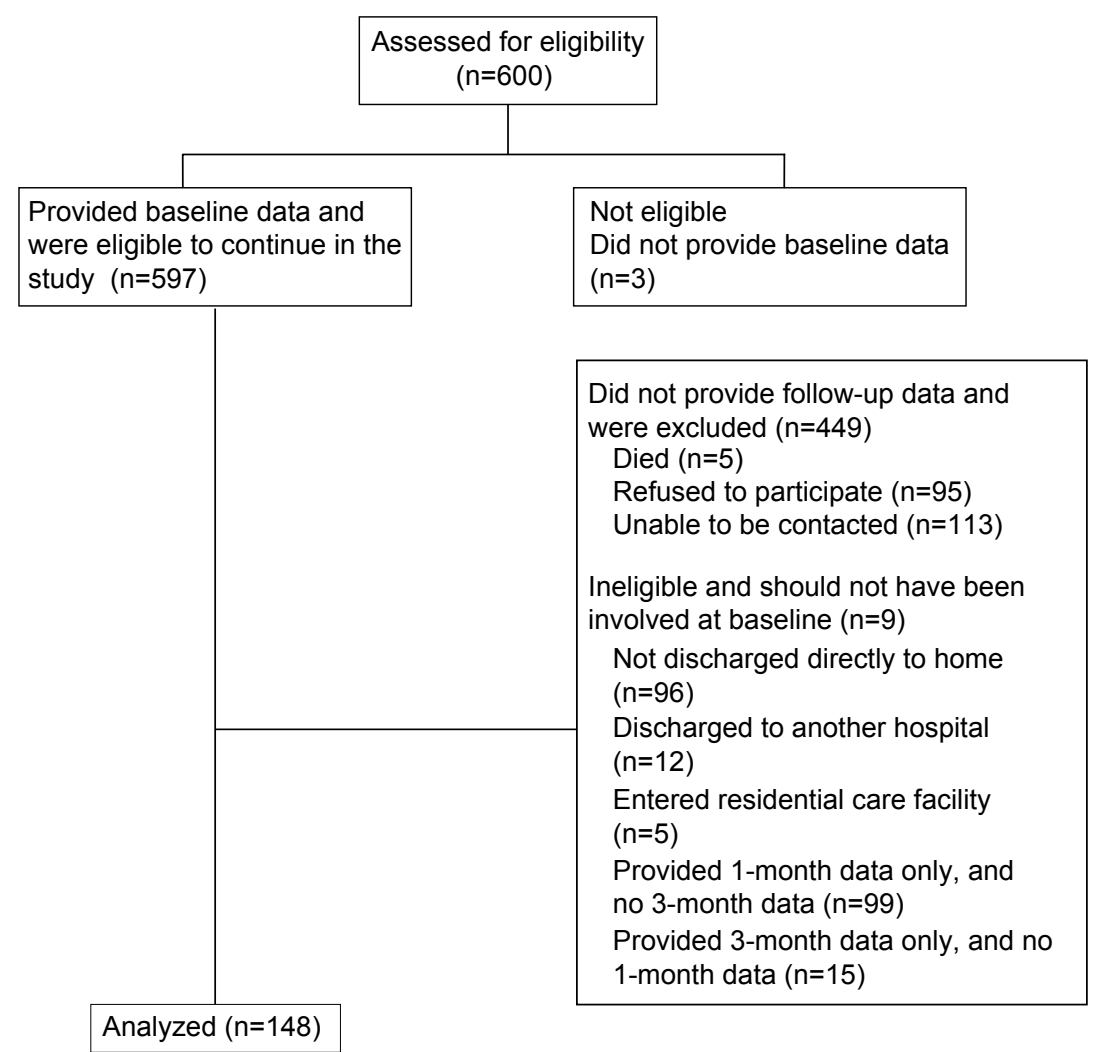

Figure 2 CONSORT diagram reporting subject attrition over the study period.

Table I Descriptive statistics of all study variables according to high and low MCSI

\begin{tabular}{|c|c|c|c|}
\hline & $\begin{array}{l}\text { Low MCS score } \\
n=74\end{array}$ & $\begin{array}{l}\text { High MCS score } \\
\mathrm{n}=74\end{array}$ & Significance \\
\hline Age, years (mean, $95 \% \mathrm{Cl}$ ) & $75.68(73.9 I-77.46)$ & 77.29 (75.56-79.02) & \\
\hline \multicolumn{4}{|l|}{ Age categories (n, \%) } \\
\hline $65-74$ years & $40(27)$ & $28(19)$ & $P>0.05$ \\
\hline $75-84$ years & $25(17)$ & $32(22)$ & $P>0.05$ \\
\hline $85+$ years & $9(6)$ & $14(9)$ & $P>0.05$ \\
\hline \multicolumn{4}{|l|}{$\operatorname{Sex}(n, \%)$} \\
\hline Male & $34(23)$ & $34(23)$ & $P>0.05$ \\
\hline Female & $40(27)$ & $40(27)$ & $P>0.05$ \\
\hline \multicolumn{4}{|l|}{ Education (n, \%) } \\
\hline No education & $4(3)$ & $0(0)$ & $P>0.05$ \\
\hline Some (or completed) primary & $18(12)$ & $19(13)$ & $P>0.05$ \\
\hline Some (or completed) secondary & $4 \mid(28)$ & $37(25)$ & $P>0.05$ \\
\hline Some (or completed) tertiary & II (7) & $16(11)$ & $P>0.05$ \\
\hline \multicolumn{4}{|l|}{ Living arrangement $(\mathrm{n}, \%)$} \\
\hline Alone & $26(18)$ & $32(22)$ & $P>0.05$ \\
\hline Spouse & $39(26)$ & $37(25)$ & $P>0.05$ \\
\hline Family (not spouse) & $5(3)$ & $5(3)$ & $P>0.05$ \\
\hline Not alone (otherwise unspecified) & $3(2)$ & $0(0)$ & $P>0.05$ \\
\hline \multicolumn{4}{|l|}{ Socioeconomic status (n, \%) } \\
\hline I (lowest) & $9(12)$ & $4(5)$ & $P>0.05$ \\
\hline 2 & $4(5)$ & 7 (9) & $P>0.05$ \\
\hline 3 & $14(19)$ & $13(18)$ & $P>0.05$ \\
\hline 4 & $17(23)$ & $16(22)$ & $P>0.05$ \\
\hline 5 (highest) & $30(4 I)$ & $34(45)$ & $P>0.05$ \\
\hline
\end{tabular}


Table I (Continued)

\begin{tabular}{|c|c|c|c|}
\hline & $\begin{array}{l}\text { Low MCS score } \\
n=74\end{array}$ & $\begin{array}{l}\text { High MCS score } \\
n=74\end{array}$ & Significance \\
\hline \multicolumn{4}{|l|}{ Primary language $(\mathrm{n}, \%)$} \\
\hline English not primary language & $14(9)$ & $60(4 I)$ & $P>0.05$ \\
\hline English is primary language & $14(9)$ & $60(4 I)$ & $P>0.05$ \\
\hline MMSE (mean, 95\% Cl) & $26.36(3.7 I)$ & $26.96(3.26)$ & $P>0.05$ \\
\hline$<27.5$ & $39(26.4)$ & $35(23.6)$ & $P>0.05$ \\
\hline$>27.5$ & $35(23.6)$ & $39(26.4)$ & \\
\hline \multicolumn{4}{|l|}{ Hospitalizations (n, \%) } \\
\hline \multicolumn{4}{|l|}{ Baseline } \\
\hline None & $56(38)$ & $49(33)$ & $P>0.05$ \\
\hline$\geq 1$ & $18(12)$ & $25(17)$ & $P>0.05$ \\
\hline \multicolumn{4}{|l|}{ I month } \\
\hline None & $50(34)$ & $60(4 I)$ & $P>0.05$ \\
\hline$\geq 1$ & $24(16)$ & $14(9)$ & $P>0.05$ \\
\hline \multicolumn{4}{|l|}{3 months } \\
\hline None & $63(43)$ & $67(45)$ & $P>0.05$ \\
\hline$\geq 1$ & II (7) & $7(5)$ & \\
\hline \multicolumn{4}{|l|}{ Falls (n, \%) } \\
\hline \multicolumn{4}{|l|}{ Baseline } \\
\hline None & $35(24)$ & $19(33)$ & $P>0.05$ \\
\hline$\geq 1$ & $39(26)$ & $25(17)$ & $\mathbf{P}<0.05$ \\
\hline \multicolumn{4}{|l|}{ I month } \\
\hline None & $65(44)$ & $71(48)$ & $P<0.05$ \\
\hline$\geq 1$ & $9(6)$ & $3(2)$ & $\mathbf{P}<0.05$ \\
\hline \multicolumn{4}{|l|}{3 months } \\
\hline None & $63(43)$ & $67(45)$ & $\mathbf{P}<0.05$ \\
\hline$\geq 1$ & II (7) & $7(5)$ & $P<0.05$ \\
\hline \multicolumn{4}{|l|}{ Use of gait aids (n, \%) } \\
\hline \multicolumn{4}{|l|}{ Baseline } \\
\hline No gait aid at the time of capture & $50(34)$ & $52(35)$ & $P<0.05$ \\
\hline Use gait aid at the time of capture & $24(16)$ & $22(15)$ & $P<0.05$ \\
\hline \multicolumn{4}{|l|}{ I month } \\
\hline No gait aid at the time of capture & $50(34)$ & $51(35)$ & $P<0.05$ \\
\hline Use gait aid at the time of capture & $24(16)$ & $23(15)$ & $P<0.05$ \\
\hline \multicolumn{4}{|l|}{3 months } \\
\hline No gait aid at the time of capture & $42(28)$ & $59(40)$ & $P<0.05$ \\
\hline Use gait aid at the time of capture & $32(22)$ & $15(10)$ & $P<0.05$ \\
\hline \multicolumn{4}{|l|}{ Community support (n, \%) } \\
\hline \multicolumn{4}{|l|}{ No community support at capture } \\
\hline Baseline & $57(39)$ & $56(38)$ & $P>0.05$ \\
\hline I month & $56(38)$ & $56(38)$ & $P>0.05$ \\
\hline 3 months & $53(36)$ & $48(32)$ & $P>0.05$ \\
\hline \multicolumn{4}{|l|}{ Used community support at capture } \\
\hline Baseline & $17(\mathrm{II})$ & $18(12)$ & $P>0.05$ \\
\hline I month & $18(12)$ & $17(11)$ & $P>0.05$ \\
\hline 3 months & $21(14)$ & $26(18)$ & $P>0.05$ \\
\hline IADL total (mean, $95 \% \mathrm{Cl}$ ) & $14.66(13.98-15.34)$ & $15.44(14.9-15.89)$ & $P<0.05$ \\
\hline I month & 14.01 (13.18-14.84) & $15.56(15.11-16.01)$ & $P<0.05$ \\
\hline 3 months & $12.6(11.64-13.57)$ & $14.16(13.67-14.65)$ & $P<0.05$ \\
\hline \multicolumn{4}{|l|}{ IADL in binary categories $(n, \%)$} \\
\hline \multicolumn{4}{|l|}{ Low } \\
\hline Baseline & $22(15)$ & $8(5)$ & $P>0.05$ \\
\hline I month & $25(17)$ & $7(5)$ & $P>0.05$ \\
\hline 3 months & $32(22)$ & $25(17)$ & $P>0.05$ \\
\hline High & & & \\
\hline Baseline & $52(35)$ & $66(45)$ & $P>0.05$ \\
\hline I month & $49(33)$ & $67(45)$ & $P>0.05$ \\
\hline 3 months & $42(28)$ & $49(33)$ & $P>0.05$ \\
\hline
\end{tabular}

Note: The significant $P$-values are shown in bold.

Abbreviations: $\mathrm{Cl}$, confidence interval; IADL, instrumental activities of daily living; MCS, mental quality of life component score; MCSI, mental quality of life component score at I month; MMSE, Mini-Mental State Examination. 
Table 2 Change in variables measured at baseline and at I month after presentation at the emergency department

\begin{tabular}{ll}
\hline & Frequency, $\mathbf{n}(\%)$ \\
\hline IADL total score & $39(28)$ \\
Improved & $73(49)$ \\
No change & $36(23)$ \\
Decreased & \\
Frequency of hospitalization(s) & $31(21)$ \\
Decreased & $91(61)$ \\
No change & $26(18)$ \\
Increased & \\
Frequency of fall(s) & $59(40)$ \\
Decreased & $86(58)$ \\
No change & $3(2)$ \\
Increased & \\
Living status & $33(22)$ \\
Now living with someone & $80(54)$ \\
No change & $35(24)$ \\
Now living alone & \\
Requiring a carer & $21(14)$ \\
Now requires a carer & $115(78)$ \\
No change & $12(8)$ \\
Not requiring a carer anymore & \\
Use of gait aids & $30(20)$ \\
Now uses gait aids & $89(60)$ \\
No change & $29(20)$ \\
Not using gait aids anymore & \\
Receipt of community support & $25(17)$ \\
Now receiving support & $98(66)$ \\
No change & $25(17)$ \\
Not receiving community support anymore & \\
\hline Abrevaton: IADL & \\
\hline
\end{tabular}

Abbreviation: IADL, instrumental activities of daily living.

in the unadjusted model (Table 4). This association was thus the only one subsequently assessed for confounding by the immutable variables. This step identified that only age exerted a significant influence on the likelihood ratio $\chi^{2}$ in this model; however, the addition of age did not adjust the odds ratio by $10 \%$ or more, thus age was not considered to be a significant confounder.

Table 3 Association between MCSI and change in mutable functional decline measures between baseline and I month post emergency department

\begin{tabular}{ll}
\hline MCSI & Unadjusted OR (95\% CI) \\
\hline Hospitalizations $(\mathrm{n})$ & $0.96(0.75-1.15)$ \\
Falls $(\mathrm{n})$ & $0.87(0.62-1.22)$ \\
IADL total score & $1.002(0.87-1.15)$ \\
Requiring a carer & $0.64(0.31-1.30)$ \\
Use of gait aids & $0.64(0.38-1.07)$ \\
Community support & $1.17(0.67-2.04)$ \\
Living status & $0.81(0.50-1.31)$ \\
\hline
\end{tabular}

Abbreviations: $\mathrm{Cl}$, confidence interval; IADL, instrumental activities of daily living; MCSI, mental quality of life component scores at I month; OR, odds ratio.

\section{Predictive capacity of MCSI and FD measures at month 3 post ED presentation}

In the unadjusted association of MCS1 and mutable FD measures 3 months post ED discharge (study question 4), only total IADL scores again showed a significant association (Table 4). This relationship was subsequently deconfounded by the mutable variables, and with regard to the cross-sectional analysis reported in study question 2, only age showed a significant change in the likelihood ratio $\chi^{2}$. Similarly, addition of age to the model did not adjust the odds ratio by $10 \%$ or more, thus age was not considered to be a significant confounder.

Figure 3 reports over-time trends of total IADL scores and the percentages of falls and hospital admissions. There is a significant decreasing linear trend regarding total IADL scores for the low MCS group ( $P=0.025)$, which highlights the potential impact of quality of life on activities of daily living. For the group with above median mental quality of life, there was no significant linear trend $(P=0.388)$. Further analysis showed that there was no significant difference among the IADL means of the high MCS group at baseline, 1 month, and 3 months post ED discharge $(P=0.156)$. However, a significant difference was identified between the means of the low MCS group taken at the three time points $(P=0.021)$.

There was no convincing linear trend over 3 months with regard to falls $(P=0.206)$ or hospitalization $(P=0.422)$ for high or low MCS1 categories. However, considering differences in falls or hospitalizations, between the high and low MCS1 categories at each time point, there was a significantly higher percentage of falls $(P=0.036)$ and hospitalizations $(P=0.039)$ at 3 months for the low MCS1 group compared with the high MCS1 group.

\section{Discussion}

This paper provides the first findings, of which we are aware, that low mental component quality of life scores after an ED discharge for a minor health crisis could be associated with decreasing function over the following 3 months. This association was not confounded by any immutable patient descriptor or by cognition at time of recruitment. Low MCS scores 1 month after ED discharge were also not strongly associated with downstream falls or hospitalizations.

This study reports on a relatively small subset of older people, and should be seen as a preliminary or scoping study for what potentially could be an important finding underpinning future research, to identify, and intervene to 
Table 4 Association between MCSI and mutable FD factors taken at I and 3 months

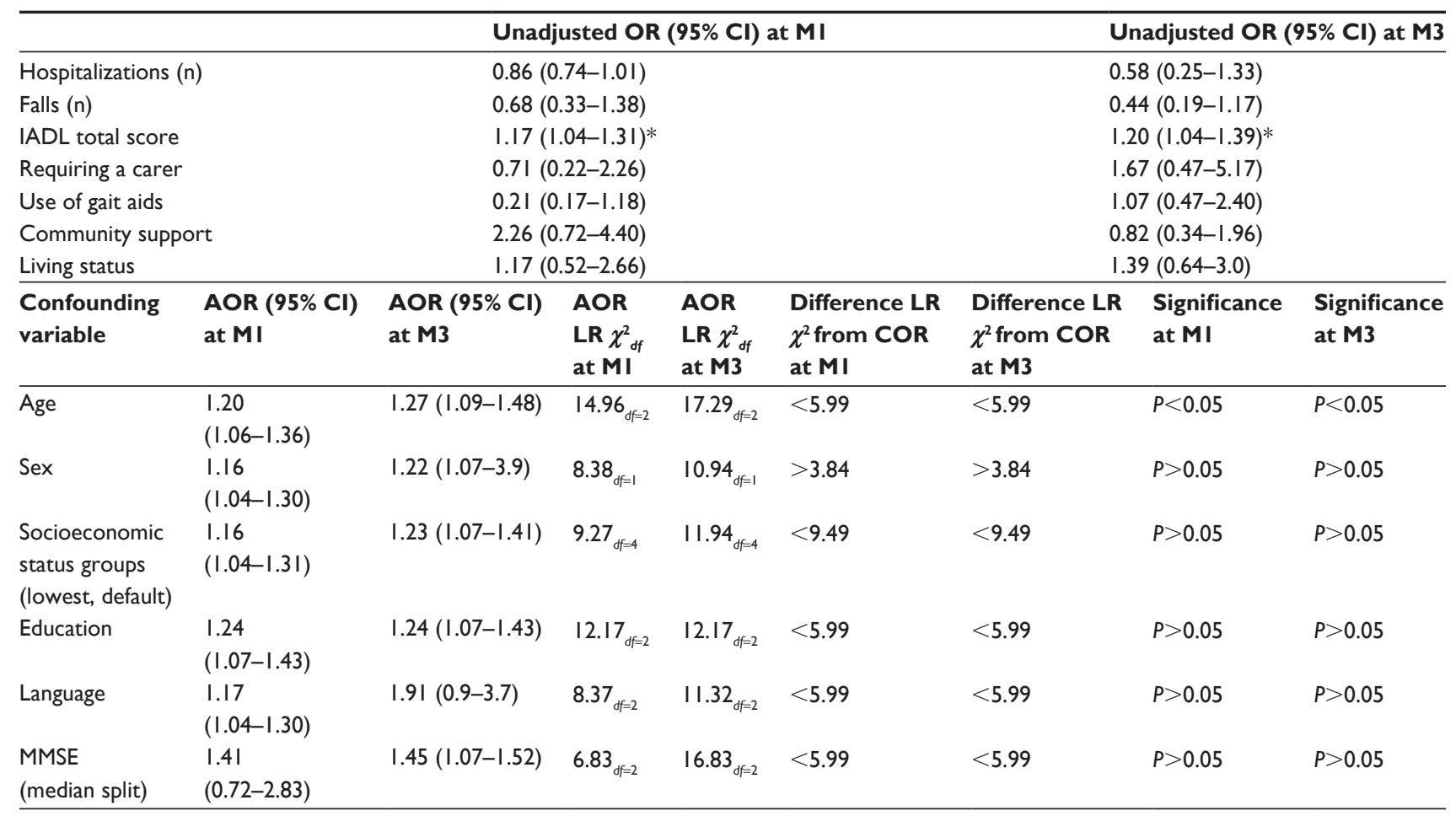

Note: *Indicates significant $P$-value, $<0.05$.

Abbreviations: MI, I month; M3, 3 months; Cl, confidence interval; AOR, adjusted odds ratio; COR, crude odds ratio; df, degrees of freedom; FD, functional decline; IADL, instrumental activities of daily living; LR, likelihood ratio; MCSI, mental quality of life component scores at I month; OR, odds ratio; MMSE, Mini-Mental State Examination.

reverse, early FD. Included in MCS calculations from the SF12 instrument are measures of vitality, social functioning, role-emotional, and mental health scales. ${ }^{20}$ It is logical that the higher the vitality, social functioning, or emotional scale scores, the higher the scores for physical activity. Several studies have reported on the association between decreased physical activity and depression or anxiety, ${ }^{28-32}$ and this paper provides evidence to consider more closely the importance of the role of mental quality of life after a minor health scare resulting in an ED presentation.

Based on trend analysis, older people with low MCS1 scores demonstrated a significant over-time decline in their total IADL scores. This validates our hypothesis that low MCS1 post ED discharge is an important feature of downstream decline in higher-order activities of daily living. It is possible that low mental quality of life has a cyclic relationship with low IADL, where one feeds on the other. This could be a manifestation of the vicious cycle in the interrelationship between depression and disability. ${ }^{33,34}$ Emotional health is responsive to treatment, and it is reasonable to expect that interventions to improve mental quality of life could also reverse early declining function. ${ }^{35}$
Those with low MCS1 scores were more likely to be hospitalized 3 months post ED discharge. This finding supports the claim by Aminzadeh and Dalziel ${ }^{36}$ regarding higher proportions of older people admitted to hospital after an ED presentation. Moreover, we found a significant difference between the number of falls in the low and high MCS1 groups at 3 months post discharge, with the low MCS group having a higher likelihood of falling. Mental status deficits are reported to be strongly related to falls. ${ }^{37,38}$

The study has several limitations. First, the use of selfreport variables such as frequency of falls and hospitalizations may incur biased responses, because awareness of these conditions may not be the same for the low and high MCS1 groups. A second limitation is the small sample size and short duration of the study, precluding inference of a direct causal relationship. Third, decreased mental quality of life scores are only summary indicators of many underlying issues, for instance confidence about living independently, fears for the future, and anxiety about the implications of health crises, however minor. Nevertheless, our findings have implications for future research regarding ways to detect early FD, and when and how to intervention to prevent or reverse declining function among the elderly. 
Total IADL scores over time

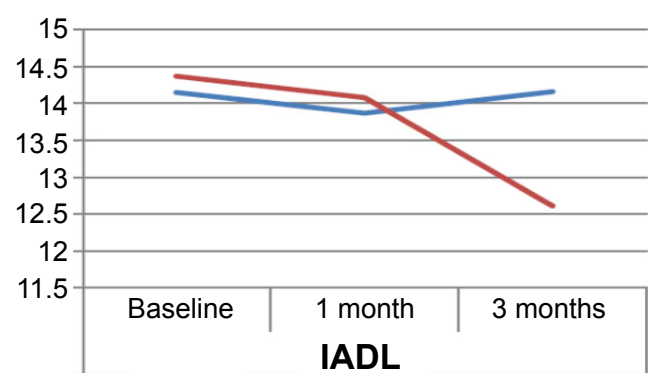

Percent falls over time

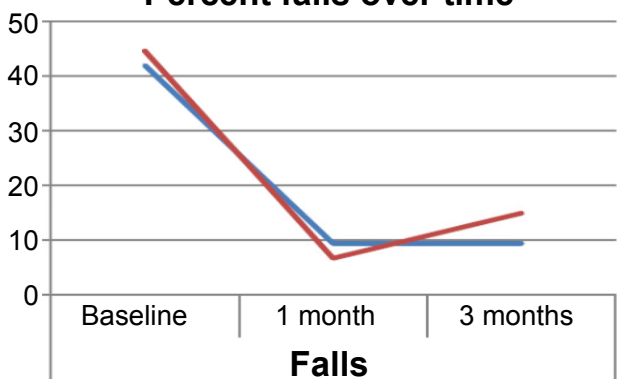

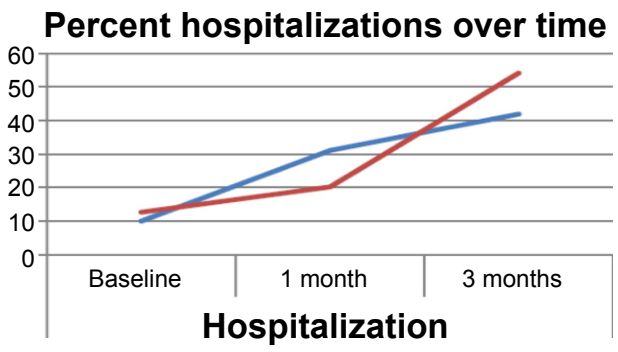

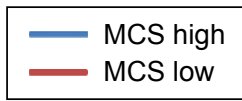

Figure 3 Total IADL scores, percentage of falls, and percentage of hospitalizations over time (baseline, I month, and 3 months post ED discharge) stratified by MCSI. Abbreviations: ED, emergency department; IADL, instrumental activities of daily living; MCS, mental quality of life component score; MCSI, mental quality of life component score at I month.

\section{Conclusion}

Our research suggests that low mental quality of life measured 1 month post ED discharge for a minor health complaint could be an important factor in early detection of FD, particularly identified in higher-order IADL, hospitalizations, and falls. Knowing this, older people could be screened for mental quality of life within a few weeks of attending ED for a minor health concern, and if they score low, early personalized interventions to improve quality of life in mental health domains could be developed, with the assumption that this may improve subsequent mental and physical functioning. Early interventions to improve vitality, well-being, depression, and/or emotional engagement could be as inexpensive as addressing individuals' concerns regarding ongoing safe community living, recommending that they join a community group or attend counseling. Providing such interventions early could translate into significant downstream public health savings, in terms of reduced hospitalizations, falls, or transition to residential care. The health benefits of early intervention to improve mental quality of life and arrest declining function could be identified within 2-3 months.

\section{Acknowledgments}

This paper was written from data collected as part of an Australian Research Council linkage grant, with funding from the Australian Research Council 2011-2012 and industry funding from the Central Northern Adelaide Health Service (via the Department of Health, South Australia). The authors would like to acknowledge the other grant investigators for their work in the Australian Research Council linkage grant, ie, John Forward, Louise Gordge, Saravana Kumar, and John Moss.

\section{Author contributions}

All authors contributed equally to the conceptualization of the study, and the final writing and preparation of the manuscript. $\mathrm{KG}$ and AA shared the statistical analysis.

\section{Disclosure}

The authors report no conflicts of interest in this work.

\section{References}

1. United Nations Department of Economic and Social Affairs, Population Division. World population aging: 1950-2050. Available from: http://www.un.org/esa/population/publications/worldageing19502050. Accessed October 31, 2013.

2. Australian Bureau of Statistics. Who are Australia's older people? Reflecting a nation: stories from the 2011 census, 2012-2013. Available from: http:// www.abs.gov.au/ausstats/abs@.nsf/Lookup/2071.0main+features7520122013. Accessed October 31, 2013.

3. Grimmer-Somers K, Beaton K, Young L, et al. Estimating risk of functional decline occurring after discharge in elderly people presenting to one large Australian public tertiary hospital emergency department. Aust Health Rev. 2013;37:341-347.

4. Caplan GA, Williams AJ, Daly B, et al. A randomized, controlled trial of comprehensive geriatric assessment and multidisciplinary intervention after discharge of elderly from the emergency department - the DEED II study. J Am Geriatr Soc. 2004;52:1417-1423.

5. Hebert R, Brayne C, Spiegelhalter D. Factors associated with functional decline and improvement in a very elderly community dwelling population. Am J Epidemiol. 1999;150:501-510. 
6. Crotty M, Giles LC, Halbert J, et al. Home versus day rehabilitation: a randomised controlled trial. Age Ageing. 2008;37:628-633.

7. de Vos AJ, Asmus-Szepesi KJ, Bakker TJ, et al. Integrated approach to prevent functional decline in hospitalized elderly: the Prevention and Reactivation Care Program (PReCaP). BMC Geriatr. 2012;12:1-12.

8. Rockwood K, Howlett SE, MacKnight C, et al. Prevalence, attributes, and outcomes of fitness and frailty in community-dwelling older adults: report from the Canadian Study of Health and Aging. J Gerontol A Biol Sci Med Sci. 2004;59A:1310-1317.

9. Gafforini S, Carson N. Primary care type presentations to public hospitals: a local in-hours and after hours population comparison. Available from: www.inwmml.org.au/fetch.cfm?fid=E8EC48CA-93BF-0411. Accessed January 3, 2015.

10. Rockwood K, Song X, MacKnight C, et al. A global clinical measure of fitness and frailty in elderly people. CMAJ. 2005;173:489-495.

11. Rockwood K, Mitnitski A. Limits to deficit accumulation in elderly people. Mech Ageing Dev. 2006;127:494-496.

12. Mehta KM, Yaffe K, Covinsky KE. Cognitive impairment, depressive symptoms, and functional decline in older people. J Am Geriatr Soc. 2002;50:1045-1050.

13. Dunn AL, Trivedi MH, O’Neal HA. Physical activity dose-response effects on outcomes of depression and anxiety. Med Sci Sports Exerc. 2001;33:S587-S597.

14. Gill S, Butterworth P, Rodgers B, MacKinnon A. Validity of the mental health component scale of the 12 item Short Form Health Survey (MCS 12) as a measure of common mental disorders in the general population. Psychiatry Res. 2007;152:63-71.

15. The Western Australian Centre for Evidence Informed Healthcare Practice, Curtin University. Triage in the emergency department. Available from: http://www.bhi.nsw.gov.au/_data/assets/pdf_file/0016/170620/ Examples_of_triage_conditions.pdf. Accessed January 8, 2015.

16. Australian Bureau of Statistics. Information paper: an introduction to Socio-Economic Indexes for Areas (SEIFA). Available from: http:// www.abs.gov.au/ausstats/abs@.nsf/mf/2039.0. Accessed October 31, 2013.

17. Lawton M, Brody E. Assessment of older people: self-maintaining and instrumental activities of daily living. Gerontologist. 1969; 9:179-186.

18. Russell M, Hill K, Blackberry I, Day LL, Dharmage SC. Falls risk and functional decline in older adults discharged directly from emergency department. J Gerontol. 2006;61:1090-1095.

19. Folstein MF, Folstein SE, McHugh PR. Mini-Mental State: a practical method for grading the cognitive state of patients for the clinician. J Psychiatr Res. 1975;12:189-198.

20. Sager MS, Rudberg MA, Jalaluddin M, et al. Hospital Admission Risk Profile (HARP): identifying older patients at risk for functional decline following acute medical illness and hospitalization. J Am Geriatr Soc. 1996;44:251-257.

21. Ware JE, Kosinski M, Keller S. A 12-Item Short-Form Health Survey: construction of scales and preliminary tests of reliability and validity. Med Care. 1996;34:220-233.
22. Ware JE. SF-36 health survey update. Spine. 2000;25(24): 3130-3139.

23. SF-36 org. US population norms 1998. Available from: http://www. sf-36.org/research/sf98norms.pdf. Accessed October 31, 2013.

24. Gill SC, Butterworth P, Rodgers B, et al. Mental health and the timing of men's retirement. Soc Psychiatry Psychiatr Epidemiol. 2006;41:515-522.

25. Sanderson K, Andrews G. The SF-12 in the Australian population: cross validation of item selection. Aust N Z J Public Health. 2007;26:343-345

26. Microsoft. Microsoft Excel. Redmond, WA, USA: Microsoft; 2010.

27. Stata version 12.1. Data Analysis and Statistical Software. College Station, TX, USA: StataCorp LP; 2012.

28. Cahn-Weiner D, Malloy P, Boyle P, Marran M, Salloway S. Prediction of functional status from neuropsychological tests in community dwelling elderly individuals. Clin Neuropsychol. 2000;14:187-195.

29. Gandek B, Ware JE, Aaronson NK, et al. Cross-validation of item selection and scoring for the SF-12 health survey in nine countries: results from IQOLA project. J Clin Epidemiol. 1998;51:1171-1178.

30. Lenze E, Rogers J, Martire L, et al. The association of late life depression and anxiety with physical disability: a review of the literature and prospectus for future research. Am J Geriatr Psychiatry. 2001;9:113-135.

31. McCuster J. A two step intervention for seniors released from hospital emergency departments. Report ISAR guide 2, 2003:1-26. 2003.

32. Yoshida I, Ihara K, Suzuki Y. Depression status as a reliable predictor of functional decline among Japanese community-dwelling older adults: a 12-year population-based prospective cohort study. Int J Geriatr Psychiatry. 2009;24:1192-1200.

33. Bruce ML, Van Citters AD, Bartels SJ. Evidence-based mental health services for home and community. Psychiatr Clin North Am. 2005; 28:1039-1060.

34. Goodwin R. Association between physical activity and mental disorders among adults in the United States. Prev Med. 2003;26:698-703.

35. Callahan K, Feiereisel K, Williamson J, et al. A three-year competency based geriatric curriculum for internal medicine residents. J Am Geriatr Soc. 2012;60:S207-S208.

36. Aminzadeh F, Dalziel W. Older adults in the emergency department: a systematic review of patterns of use, adverse outcomes and effectiveness of interventions. Ann Emerg Med. 2002;39:238-247.

37. Hook M, Devine E, Lang N. Using a computerized fall risk assessment process to tailor interventions in acute care. Available from: http://www. ahrq.gov/professionals/quality-patient-safety/patient-safety-resources/ resources/advances-in-patient-safety-2/vol1/advances-hook_25.pdf. Accessed October 31, 2013.

38. Shumway-Cook A, Brauer S, Woolacott M. Predicting the probability for falls in community-dwelling older adults using the timed up and go test. Phys Ther. 2000;80:896-902.
Clinical Interventions in Aging

\section{Publish your work in this journal}

Clinical Interventions in Aging is an international, peer-reviewed journal focusing on evidence-based reports on the value or lack thereof of treatments intended to prevent or delay the onset of maladaptive correlates of aging in human beings. This journal is indexed on PubMed Central, MedLine,
Dovepress

CAS, Scopus and the Elsevier Bibliographic databases. The manuscript management system is completely online and includes a very quick and fair peer-review system, which is all easy to use. Visit http://www.dovepress. com/testimonials.php to read real quotes from published authors. 